\title{
Gastroparesis following endoscopic submucosal dissection for early gastric cancer
}

A 67-year-old man with no history of chronic disease was referred to our hospital because of progressive abdominal distention and poor appetite in September 2009. He had undergone endoscopic submucosal dissection (ESD) at our endoscopy center for a large, flat lesion in the gastric incisure 2 months previously. The lesion had been a type IIb with a diameter of $10 \mathrm{~cm}$. It had been resected in one piece and was shown histologically to be an early gastric cancer with tumor-free lateral and vertical margins. Following his discharge, after 1 week the patient had begun to experience abdominal bloating and early fullness after only a few bites of food associated with reflux. The symptoms had deteriorated progressively over the subsequent 2 months, accompanied by weight loss of about $2.5 \mathrm{~kg}$.

At endoscopy he was found to have gastric retention and a large ulcer at the site of the previous resection, but there was no evidence of gastric outlet obstruction. A barium radiographic study showed poor gastric peristalsis. He was treated with enteral feeding and gastrointestinal decompression, with oral administration of erythromycin, prokinetic agents, and anti anxiety medications all used to improve stomach emptying. About 6 weeks later, a normal eating pattern had been restored and repeat radiographic examination demonstrated near-normal motility. The patient was successfully discharged in November 2009. Repeat endoscopy 2 months after this discharge revealed a scar at the original wound location and he remained symptom-free.

In this case, considering that there was no medical history of other diseases such as diabetes, we hypothesized that the possible mechanisms of gastroparesis were as follows. First, the size of the lesion was as large as $10 \mathrm{~cm}$, so the vagal nerves that regulate fundal relaxation and antral contraction may have been damaged during the ESD procedure. Second, local edema

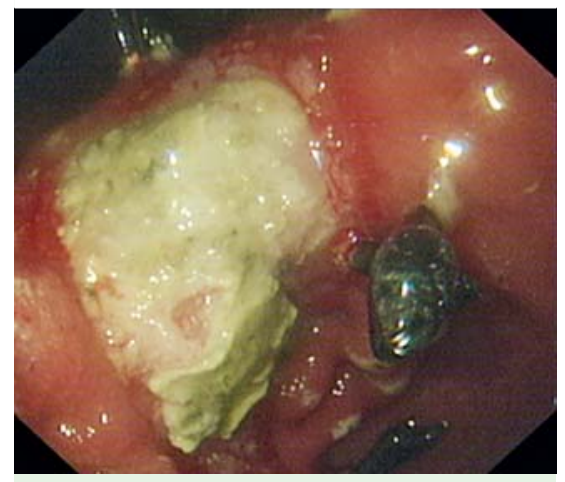

Fig. 1 Endoscopic view at the time the patient was admitted with gastric stasis showing the unhealed ulcer at the site of the previous resection.

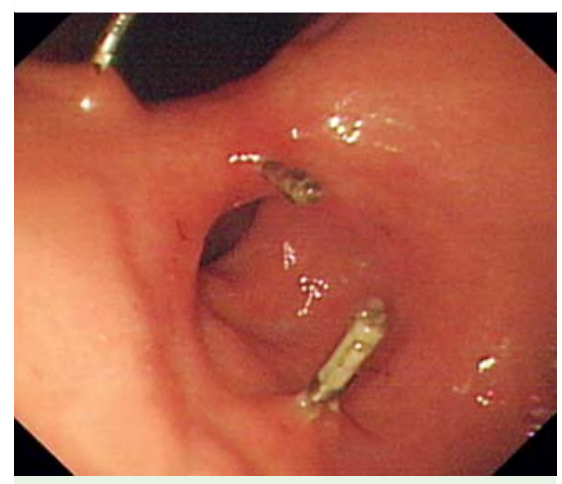

Fig. 2 Endoscopic view 2 months after his final discharge showing the presence of a scar at the original resection site.

and adhesions close to the wound may have affected the gastric motor function. Third, the patient was very anxious about the ESD procedure, so the gastrointestinal reflex inhibition may have been prolonged and gastric empting reduced. A combination of these three causes led ultimately to the delayed gastric emptying.

Endoscopy_UCTN_Code_CPL_1AH_2AZ

Competing interests: None
J. Lian ${ }^{1}$, S. Chen ${ }^{1,2}$, Y. Zhang ${ }^{2}$, L. Yao²

1 Department of Gastroenterology, Zhongshan Hospital, Fudan University, Shanghai, China

2 Endoscopy Center, Zhongshan Hospital, Fudan University, Shanghai, China

Bibliography

DOI $10.1055 / \mathrm{s}-0030-1256684$

Endoscopy 2011; 43: E369

(c) Georg Thieme Verlag KG Stuttgart · New York . ISSN 0013-726X

Corresponding author

S. Chen, MD

Department of Endoscopy Center Zhongshan Hospital

Fudan University

180 Fenglin Road

Shanghai

China

Fax: +86-21-64437583

syaochen@163.com 